\title{
Diagnose der Obstipation bei Kindern
}

\author{
Frédérique Sauvat \\ Department of Pediatric Surgery, UFR Necker-Enfants Malades, University René Descartes Paris V, Paris, France
}

\section{Schlüsselwörter \\ Obstipation • Morbus Hirschsprung • Pädiatrie}

\section{Zusammenfassung}

Die Obstipation ist ein häufiger Grund für den Besuch beim Kinderarzt. Tritt die Obstipation als Symptom auf, so können ihr, je nach Häufigkeit, Art und Schweregrad, diverse Ätiologien zugrunde liegen. Die Obstipation kann sowohl auf einer medizinischen Ursache beruhen, chirurgisch bedingt oder auch funktioneller Natur sein. Zu den Ätiologien, die einen chirurgischen Eingriff erfordern, zählt in erster Linie der Morbus Hirschsprung. Diese angeborene Erkrankung tritt zwar $50 \mathrm{Mal}$ seltener auf als die idiopathische oder funktionelle Obstipation, ist aber die wichtigste Differentialdiagnose. Die Diagnose erfolgt am besten mittels gründlicher Anamneseerhebung und sorgfältiger klinischer Untersuchung, zu der u.a. auch eine anale Untersuchung zählt. Weitere Untersuchungen sind in den meisten Fällen nicht erforderlich und sämtliche Ermittlungen sollten gemäss klinischer Überlegungen gehandhabt werden.

Copyright $\odot 2008$ Nestec Ltd., Vevey/S. Karger AG, Basel

Drei Prozent aller Besuche beim Kinderarzt und über $25 \%$ aller Besuche beim Gastroenterologen erfolgen aufgrund von Obstipation [1, 2]. Die erste Schwierigkeit beim Thema Obstipation besteht in der Definition, welche Stuhlfrequenz bei einem Kind normal ist. Tatsäch- lich variiert die Stuhlfrequenz je nach Alter und Art der Nahrung. Von Obstipation spricht man bei einer Stuhlfrequenz von weniger als drei Stühlen pro Woche. Die Obstipation kann von anderen Symptomen (Bauchschmerzen) begleitet werden, die sich mehr oder weniger störend auf den Patienten und dessen soziales Leben und den Schulbesuch auswirken [3]. Tritt die Obstipation als Symptom auf, so können ihr je nach Häufigkeit, Art und Schweregrad diverse Ätiologien zugrunde liegen [4]. Diese Ätiologien lassen sich in medizinische und chirurgische Ursachen aufteilen, wobei die Obstipation jedoch in den meisten Fällen idiopathischer bzw. funktioneller Natur ist [5].

\section{Funktionelle Obstipation}

Die bei weitem häufigste Form der Obstipation ist die funktionelle Obstipation [6]. Mit zunehmendem Alter kommt es im Darm und Dickdarm von Kindern zu normalen physiologischen Veränderungen und die Stuhlfrequenz sinkt von durchschnittlich 2,2 Stühlen pro Tag bei Säuglingen im Alter von unter einem Jahr auf durchschnittlich 1,4 Stühle bei Einjährigen [7]. In den ersten Lebensmonaten spricht man bei weniger als einem Stuhlgang pro Tag und bei älteren Kindern bei weniger als drei Stühlen pro Woche von Obstipation. Der Altersgipfel der Obstipation liegt in dem Alter, in dem bei Kindern das Toilettentraining durchgeführt wird (2-4 Jahre), wobei

\section{KARGER \\ Fax +4161306 1234 \\ E-Mail karger@karger.ch}

www.karger.com (c) 2008 Nestec Ltd., Vevey/S. Karger AG, Basel

0250-9652/07/0652-0063\$23.50/0

Online-Zugriff auf:

www.karger.com/and
Dr. F. Sauvat

Pediatric Surgery, Hôpital Necker-Enfants Malades

149 , rue de Sèvres

FR-75015 Paris (France)

Tel. +33 1444941 54, Fax +33 1444942 00, E-Mail frederique.sauvat@nck.aphp.fr 
die Prävalenz bei Jungen höher ist als bei Mädchen. Die Diagnose der funktionellen Obstipation erfolgt nach den Rom-Kriterien, wobei das Alter des Patienten zu berücksichtigen ist $[2,8]$.

Die Diagnose gilt als bestätigt, wenn mindestens zwei der folgenden Symptome länger als einen Monat andauern: (1) eine Stuhlfrequenz von höchstens zwei Stühlen pro Woche; (2) Stuhlinkontinenz mindestens einmal pro Woche; (3) Stuhlretention in der Anamnese; (4) Bauchschmerzen; (5) Fäkalom (Stuhlstein) im Rektum, und (6) Vorliegen grosser, harter Stuhlmassen, welche die Toilette verstopfen können.

Bei ungefähr 40\% der Kinder mit funktioneller Obstipation entwickeln sich die Symptome im ersten Lebensjahr. Ferner nimmt man an, dass die Prognose bei Kindern unter vier Jahren besser ist, wenn die Behandlung bereits im Alter von unter zwei Jahren begonnen wurde. Bei 84\% der Patienten kommt es ausserdem zu Enkopresis [9]. In typischen Fällen bedarf es keiner weiteren Untersuchung. Sollte sich die körperliche Untersuchung als schwierig erweisen (z.B. bei Adipositas), so reicht eine normale Röntgenuntersuchung des Abdomens zur Bewertung der Stuhlretention [10]. Erkrankungen der Wirbelsäule bzw. des Rückenmarks müssen durch eine entsprechende Untersuchung ausgeschlossen werden (siehe unten). Ebenso muss mit dem Finger eine digitale Untersuchung des Anus und des Rektums durchgeführt werden, um eine eventuelle minimale anorektale Fehlbildung auszuschliessen, den Muskeltonus des Analsphinkters zu untersuchen und das Vorliegen eines Fäkaloms zu überprüfen [11]. Die Behandlung besteht in einer möglichst vollständigen Entleerung des Dickdarms, einer Änderung der Stuhlkonsistenz (d.h. weicheren Stühlen) und einer Änderung der Ernährung und Lebensgewohnheiten. Ernährungsfehler (Dehydratation, ballaststoffarme Ernährung etc.) können ebenso wie lokale Ursachen zu einer persistierenden Obstipation mit schwieriger oder schmerzhafter Stuhlentleerung führen.

\section{Medizinische Ursachen der Obstipation}

In selteneren Fällen beruht die Obstipation im Kindesalter auf einer endokrinen Störung oder einer metabolischen oder toxischen Ursache.

Die wichtigste endokrine Ursache der Obstipation ist die Hypothyreose. Dank der gezielten Vorsorgeuntersuchung von Neugeborenen (Guthrie-Test) wird diese Erkrankung inzwischen jedoch frühzeitig diagnostiziert, so dass die typischen neurologischen Folgen einer späteren Diagnose gemindert werden. Auch Diabetes [12] und Hypoparathyreoidismus wurden als Ursachen der Obstipation beschrieben, wobei Störungen der Darmpassage jedoch selten als erstes Anzeichen auftreten.

Ein Elektrolytungleichgewicht kann, wie z.B. bei Hypo- und Hyperkalzämie, die Darmperistaltik herabsetzen $[13,14]$.

Auch die Möglichkeit einer Vergiftung muss bei der Anamneseerhebung stets in Betracht gezogen werden, wobei auch die eventuell von der stillenden Mutter eingenommenen Medikamente zu berücksichtigen sind. $\mathrm{Zu}$ den wichtigsten Arzneistoffen, die eine Obstipation im Säuglingsalter hervorrufen können, zählen u.a. Anticholinergika, krampflösende Mittel (Antispasmodika) und bestimmte Harze (Cholestyramin). Der mögliche Zusatz eines Andickungsmittels zur Säuglingsnahrung (Gumilk ${ }^{\circledR}$ ) muss ebenfalls in Betracht gezogen werden, da dies Obstipation oder aufgrund der Ansammlung von kompakten Stühlen, insbesondere im Zökum, sogar ein richtiges Obstruktionssyndrom verursachen kann.

Obstipation kann, wie bereits erwähnt, auch durch falsche Ernährung hervorgerufen werden. Zu den wichtigsten Ätiologien zählen: (1) Mangelernährung, Anorexie; (2) Dehydratation [15]; (3) zu viel Kuhmilch [16] und (4) insbesondere bei älteren Kindern Ballaststoffmangel.

Auch die Zöliakie [17] (Glutenunverträglichkeit) kann mit Episoden der Obstipation einhergehen.

Bei der Mukoviszidose (zystischen Fibrose) kann Obstipation in der Neugeborenenphase (Mekonium-Ileus) oder später im Säuglingsalter aufgrund einer exokrinen Bauchspeicheldrüseninsuffizienz auftreten. Ein pränatal festgestelltes Obstruktionssyndrom mit Hyperechogenität des Darmes legt den Verdacht auf Mukoviszidose nahe (wobei die endgültige Diagnose mittels Amniozentese erfolgt). Bei einem Mekonium-Ileus nach der Geburt handelt es sich um eine mit verzögertem Mekoniumabgang und abdomineller Distension einhergehende Blockade des Ileums. Auf dem Röntgenbild stellt sich der Mekonium-Ileus typischerweise als Schatten im rechten Unterbauch dar. Bei rektaler Applikation des Kontrastmittels Gastrografin zeigt sich ein atones, nicht funktionierendes Kolon und typische kleine Mekoniumpfropfe im Zökum und Ileum. Bei einer Intervention, die es unter allen Umständen zu vermeiden gilt, sieht man mit blossem Auge das charakteristische zähe Mekonium. Was die Hypothyreose anbetrifft, so ist die Vorsorgeuntersuchung in Frankreich bei Neugeborenen inzwischen Pflicht. 


\section{Chirurgische Ursachen der Obstipation}

Die wichtigste organische Ursache der Obstipation ist Morbus Hirschsprung (HD), wobei nicht vergessen werden darf, dass Morbus Hirschsprung 50 Mal seltener vorkommt als die funktionelle Obstipation.

\section{Morbus Hirschsprung}

Morbus Hirschsprung, auch als kongenitales Megakolon bezeichnet, ist eine angeborene Fehlbildung, die auf einem aganglionären Darmsegment variabler Länge beruht. Unabhängig von der Länge des betroffenen Darmsegments beginnt die intrinsische Innervation des Darms immer oberhalb des Analbereichs und erstreckt sich mehr oder weniger bis zum Dickdarm oder sogar Dünndarm. In 75-80\% der Fälle betrifft Morbus Hirschsprung das Rektum und Sigmoid [18]. In 10\% der Fälle ist der gesamte Dickdarm betroffen (totale Kolonaganglionose) und bei weniger als $1 \%$ der Patienten liegt eine totale intestinale Aganglionose (die auch das Jejenum mit einschliesst) vor. Die Anomalie beruht auf dem Fehlen der Ganglienzellen im Meissner- und Auerbach-Plexus und wird mit einer Hypertrophie der Nervenfasern assoziiert. Diese Aganglionose ist für die Hypertonizität des kranken Darms verantwortlich.

\section{Die Epidemiologie von Morbus Hirschsprung}

Morbus Hirschsprung tritt in der Allgemeinbevölkerung mit einer Häufigkeit von einer von 5000 Geburten auf [19], wobei die kurze (auf das Rektosigmoid beschränkte) Form der Erkrankung viermal häufiger bei männlichen Säuglingen auftritt. Das Risiko, dass die Erkrankung an Verwandte ersten Grades vererbt wird, ist niedrig (4\%), steigt jedoch signifikant, wenn der Indexfall weiblich ist oder unter einer «langstreckigen Form» der Erkrankung leidet, d.h. einer Form, bei der ein langes Darmsegment betroffen ist. Die meisten Fälle sind sporadisch. Nur 6 bis 15\% der Fälle sind vererbt, wobei die Vererbung autosomal-dominant mit unvollständiger Penetranz erfolgt [20-22].

\section{Klinische Präsentation}

Die Symptome variieren je nach Alter und Ausmass der Erkrankung. Eine funktionelle Obstruktion liegt in allen Fällen vor.

Diagnose von Morbus Hirschsprung bei

Neugeborenen ohne Komplikationen [23, 24]

Die Möglichkeit des Morbus Hirschsprung wird generell bei allen Neugeborenen mit funktioneller Obstrukti- on vermutet, insbesondere wenn das Neugeborene termingerecht geboren wurde und die pränatale Ultraschalluntersuchung normal war.

$\mathrm{Zu}$ den normalen klinischen Symptomen zählen: (1) verzögerter Mekoniumabgang nach 24-48 Stunden; (2) ein in den ersten zwei Lebenstagen zunehmend aufgetriebenes Abdomen und (3) Erbrechen von Nahrung, wohingegen das Erbrechen von Galle häufig erst nach einer gewissen Verzögerung auftritt.

Bester klinischer Hinweis für die Diagnose des Morbus Hirschsprung ist der explosionsartige Abgang von Gas und Mekonium nach der digitalen Untersuchung des Anus und des Rektums bzw. nach Platzierung eines Darmrohrs. Bei der auf das Rektosigmoid begrenzten Form wird das Darmrohr oberhalb des aganglionären Darmsegments platziert und ermöglicht so die Ausscheidung der zurückgehaltenen Faeces und einen schnellen Rückgang der abdominellen Distension [25]. Vor Platzierung des Rohrs ist ein Pneumoperitoneum mittels Röntgenuntersuchung auszuschliessen. Das Rohr muss äusserst vorsichtig platziert werden, um, insbesondere bei Verdacht auf Enterokolitis, eine Perforation des Darms $\mathrm{zu}$ vermeiden. Die «langstreckige Form» des Morbus Hirschsprung kann auch dann nicht ausgeschlossen werden, wenn der explosionsartige Abgang von Gas und Mekonium fehlt. In den meisten Fällen wird die Krankheit in den ersten drei Lebensmonaten diagnostiziert [26].

Die Diagnose von Morbus Hirschsprung bei

Säuglingen

Bei Neugeborenen mit Obstipation muss stets die Diagnose Morbus Hirschsprung in Betracht gezogen werden, insbesondere wenn die Motilitätsstörung nach dem Abstillen oder nach Einführen von Beikost auftritt oder zunimmt, d.h. also in einer Zeit, in der die Stühle fester werden. Die Stuhlfrequenz ist variabel, wobei die Stuhlentleerung manchmal mehrere Tage lang ausbleiben kann. Das Abdomen ist chronisch aufgetrieben, wobei sich der Zustand nach Stimulation (Laxantien etc.) bessert. Die rektale Untersuchung dient zum Ausschluss anorektaler Fehlbildungen (lokale Analstenose) und hilft, eine Stuhlimpaktion im oberen Darmbereich zu entdecken. Das Wachstum ist in der Regel schlecht.

\section{Diagnose zu einem späteren Zeitpunkt}

Die kurze Form der HD kann in fast allen Fällen diagnostiziert werden. Die Kinder weisen eine lange Anamnese mit hartnäckiger Obstipation auf, die mit rezidivierenden Episoden eines Beinahe-Verschlusses bzw. mit Distension der gesamten Abdominalwand einhergeht. 

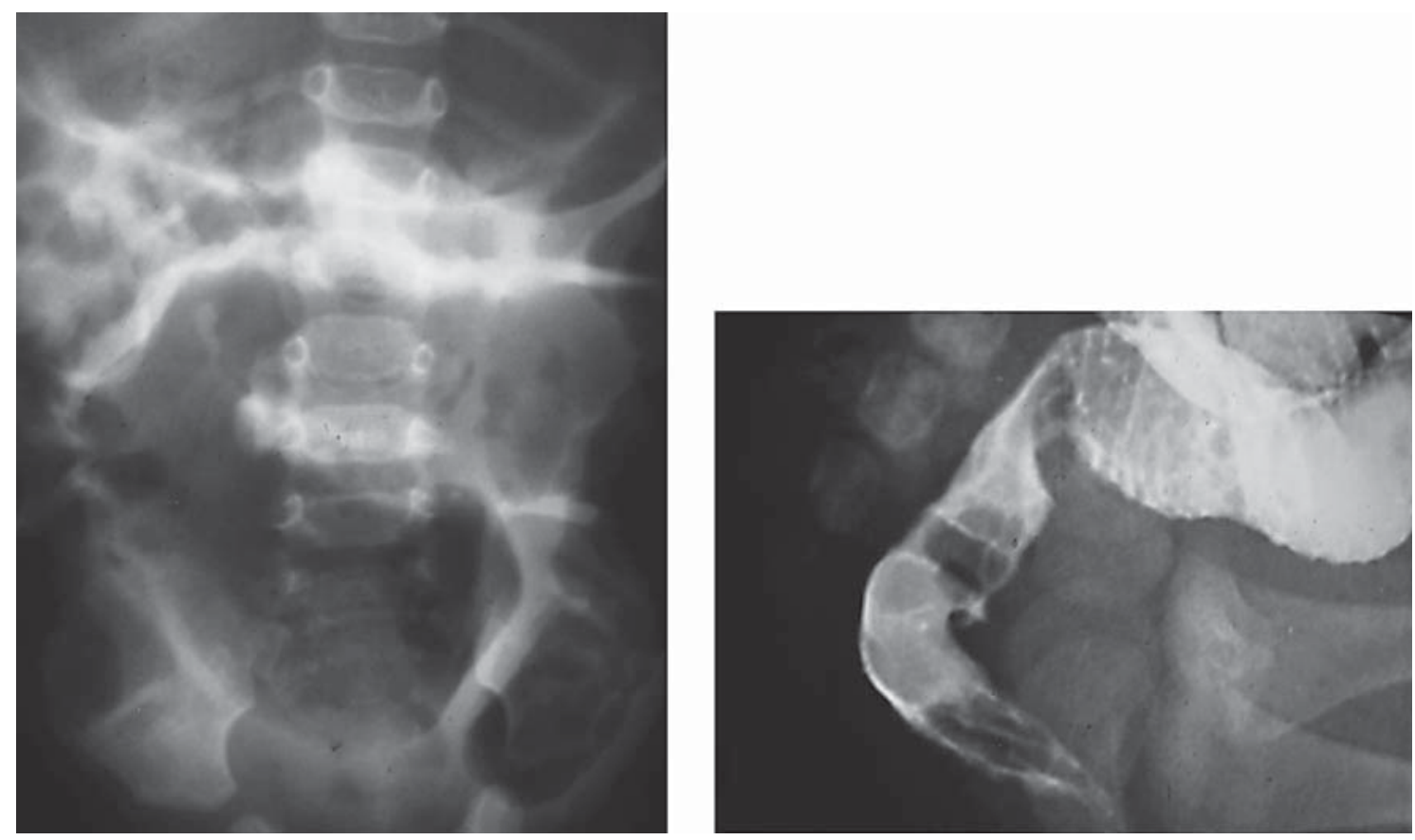

Abb. 1. Typisches Röntgenbild bei Morbus Hirschsprung im Rektumsigmoid mit Barium-Kontrastmittel.

Die Obstipation ist ab Geburt bekannt und eine Stuhlentleerung findet nur alle paar Tage nach Stimulation statt. Ausgetrocknete Stühle sind dabei häufig mit flüssigen oder, aufgrund der chronischen Retention, übelriechenden Stühlen vermischt. Ein wichtiger klinischer Aspekt ist, dass die Kontinenz bei Hirschsprung-Patienten immer normal ist. Enkopresis wird nicht mit Morbus Hirschsprung in Verbindung gebracht. In einigen Fällen wirkt sich der Morbus Hirschsprung signifikant auf das Wachstum und die langfristige psychische Entwicklung aus [27].

Die «langstreckige Form» des Morbus Hirschsprung $[28,29]$

Die Diagnose der langstreckigen Form des Morbus Hirschsprung ist oft schwierig und erfolgt häufig erst mit einer gewissen Verzögerung [30]. Das klinische Bild der Erkrankung variiert und kann folgende Formen annehmen:(1) ein (manchmal unvollständiger) Darmverschluss beim Neugeborenen mit oder ohne spontanen Mekoniumabgang, wobei klinische Untersuchungen, wie z.B. eine Röntgenuntersuchung, zur Diagnose eines Mekonium-Ileus beitragen können; (2) eine Enterokolitis bei Neugeborenen [31, 32] könnte, bei Ausschluss einer Frühgeburt oder schweren Herzerkrankung, ebenfalls mit der langstreckigen Form des Morbus Hirschsprung zusammenhängen; (3) eine akute Form der Erkrankung mit Durchbruch der Appendix oder Zökums [33], und (4) eine, insbesondere aufgrund einer Unverträglichkeit, schwere Obstipation bei Neugeborenen mit stärkeren Auswirkungen auf das Wachstum.

\section{Morbus Hirschsprung mit Komplikationen}

Die zwei Hauptkomplikationen bei Morbus Hirschsprung sind Darmperforation und Enterokolitis. Diese Komplikationen können entweder zur Diagnose Morbus Hirschsprung führen oder im Verlauf dieser Erkrankung auftreten und lebensbedrohlich sein.

Die Enterokolitis [34] kann als Septikämie intestinalen Ursprungs betrachtet werden, die entsteht, wenn sich die Mikroben im Darmlumen aufgrund der Stasis in Verbindung mit einer durch die abdominelle Distension hervorgerufenen Ulzeration der Darmwand und Ischämie vermehren. Klinisch zeigt sich eine ausgeprägte schmerzhafte Distension des Abdomens und eine schwere Sepsis. Die Stühle sind flüssig und faulriechend oder blutig. Das Röntgenbild zeigt eine allgemeine Dilatation, wobei eine Pneumatose jedoch selten ist. Bei Morbus Hirschsprung-Patienten erfolgt die Behandlung der Enterokolitis mittels Breitbandantibiotikum (gegen die 
grammnegativen und anaeroben Keime) und operativer Umleitung (in eine normal innervierte Zone).

In einer normal innervierten Zone kann es zur Darmperforation kommen [35]. Bei Pneumoperitoneum muss eine Notoperation durchgeführt werden. Die eindeutige Diagnose der Hirschsprung-Krankheit ist möglicherweise schwierig, da ein Kalibersprung fehlt. In einem solchen Fall sollte an die Diagnose Morbus Hirschsprung gedacht, oberhalb der Darmperforation ein Stoma angelegt und eine Rektumbiopsie durchgeführt werden [36].

\section{Die Diagnose von Morbus Hirschsprung}

Die Diagnose von Morbus Hirschsprung beruht auf Kolon-Kontrastmitteleinlauf, hauptsächlich jedoch auf einer Rektumbiopsie, da das Fehlen der Ganglienzellen nur so eindeutig diagnostiziert werden kann.

\section{Radiologische Untersuchung mit}

Kontrastmitteleinlauf [37, 38]

Ziel der radiologischen Untersuchung mit Kolon-Kontrastmitteleinlauf ist die Darstellung des Kalibersprungs zwischen dem verengten aganglionären Darmsegment und dem erweiterten gesunden Darmabschnitt. Die Untersuchung ermöglicht ferner die Längenbestimmung des aganglionären Segments. Bei Neugeborenen ist diese Untersuchung jedoch häufig schwierig zu interpretieren.

Die radiologische Untersuchung mit Kolon-Kontrastmitteleinlauf muss unter guten Bedingungen durchgeführt werden, wobei weder ein Pneumoperitoneum noch eine Enterokolitis vorliegen dürfen. Der Verlauf des Kontrastmittels (vorzugsweise Barium) wird mit einem Endoskop in Frontal- und Seitenansicht überwacht, wobei das Hauptaugenmerk auf dem Kalibersprung liegt. Standardröntgenaufnahmen (nach ca. 1 Stunde) liefern häufig die besten Ergebnisse.

Bei der auf das Rektosigmoid beschränkten Form folgt auf das Rektum, das auf dem Röntgenbild normal erscheint, eine Übergangszone, die sich bis zum Durchmesser des gesunden Dickdarmabschnitts weitet. Dieser Kalibersprung ist typisch für den Morbus Hirschsprung (Abb. 1).

Die totale Kolonaganglionose kann sich in der radiologischen Untersuchung mit Kolon-Kontrastmitteleinlauf unterschiedlich darstellen [39]: (1) als kurzes Kolon in Form eines Fragezeichens, bei dem die rechten und linken Kolon-Flexuren verschwinden (Abb. 2); (2) als Mikrokolon, das mit einer mehr oder weniger ausgeprägten Erweiterung des Ileums einhergeht - die Differenzialdiagnosen bei dieser Form sind Mekonium-Ileus oder Me-

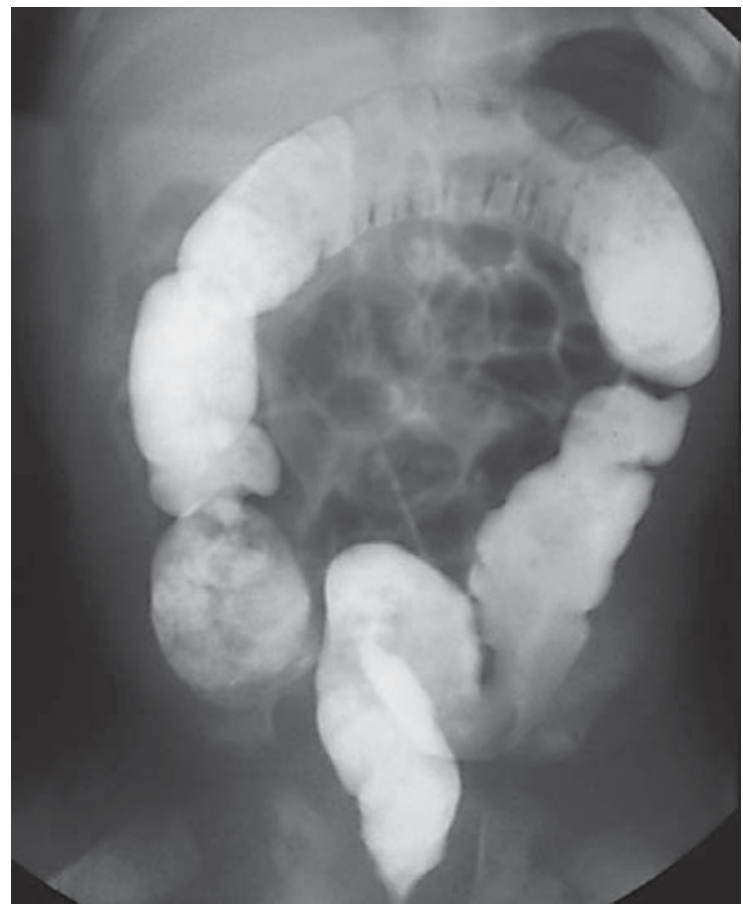

Abb. 2. Röntgenbild mit Kolon-Kontrastmitteleinlauf bei totaler Kolonaganglionose.

gazystis-Mikrokolonsyndrom, und (3) als normal aussehender Darm (30\%).

\section{Anorektale Manometrie [40, 41]}

Hauptsymptom für die Diagnose Morbus Hirschsprung ist das Fehlen der Relaxation des Sphinkters internus bei der Manometrie. Da jedoch auch falsch positive Befunde möglich sind, muss stets eine Rektumbiopsie durchgeführt werden. Man geht in der Regel davon aus, dass eine Manometrie bei Neugeborenen (jünger als drei Monate) nicht besonders sinnvoll ist.

\section{Rektumbiopsie}

Die histologische Untersuchung ermöglicht eine eindeutige Diagnose und ist daher entscheidend. Die Rektumbiopsie muss mindestens $3 \mathrm{~cm}$ oberhalb des Analrands durchgeführt werden, um ein falsch positives Ergebnis zu vermeiden (wird die Biopsie zu tief angesetzt, könnte sie in ein schon an sich hypoganglionäres Darmsegment fallen). Für die Biopsie stehen zwei Verfahren zur Verfügung:

(1) Die rektale Saugbiopsie (mit H. Noblett-Sonde) wird ohne Betäubung durchgeführt. Normalerweise nimmt man zwei Biopsieproben, einmal $3 \mathrm{~cm}$ und einmal 
$6 \mathrm{~cm}$ vom Analrand. Einschränkend ist bei dieser Methode, dass ein geschulter Pathologe benötigt wird und dass die Gewebeproben Teile der Muscularis mucosa und möglichst auch der Submucosa enthalten müssen.

(2) Die chirurgische Biopsie in Form einer Ganzwandbiopsie des Rektums (Swenson-Methode) muss zwar unter allgemeiner Anästhesie durchgeführt werden, ermöglicht aber eine tiefere und dadurch aufschlussreichere Biopsie.

Histologische Befunde, die für die Diagnose Morbus Hirschsprung sprechen, sind [42]: (1) das Fehlen der Ganglien und die Hypertrophie der Nervenfasern (die bei Neugeborenen häufig fehlen) auf fixierten Schnitten und in der Standardhistologie [43], sowie (2) die Hyperplasie der cholinergen Fasern in Teilen der Submucosa und Muskulatur in Kombination mit Kletterfasern in der Mucosa bei der immunhistochemischen Untersuchung gefriergetrockneter Schnitte.

\section{Behandlung}

Die Behandlung von Morbus Hirschsprung erfolgt durch einen chirurgischen Eingriff, bei dem das von der Aganglionose betroffene Segment entfernt und eine koloanale Anastomose zwischen dem Anus und dem normal innervierten Darm angelegt wird [44]. Vor der Operation muss die Stuhlentleerung entweder mittels rektaler Entleerung oder mittels Stoma erfolgen.

\section{Präoperative Behandlung}

Bei der rektalen Entleerung erfolgt die Stuhlentleerung mittels eines Darmrohrs, das bis oberhalb des von der Aganglionose betroffenen Darmabschnitts eingeführt wird. Das Darmohr muss ein- bis dreimal täglich appliziert werden, um einen Rückgang des aufgetriebenen Abdomens zu erzielen und die Wachstumskurve $\mathrm{zu}$ korrigieren. Diese einfache Methode kommt nur bei der kurzen Form des Morbus Hirschsprung in Frage und ermöglicht es den Patienten, zuhause auf den Operationstermin zu warten, vorausgesetzt die Familie kann diese Behandlung vornehmen und merkt, wenn keine ausreichende rektale Entleerung stattgefunden hat oder das Abdomen aufgetrieben ist.

\section{Stoma}

Hier gilt die Regel, dass das Stoma in einem normal innervierten Darmabschnitt und so distal wie möglich angelegt werden sollte, um eine Übergangszone zu vermeiden. Das Stoma wird beim letzten chirurgischen Eingriff durchgezogen. Indikationen, die zum Zeitpunkt der Diagnose für die Anlage eines Stomas sprechen, sind: «langstreckige Form» des Morbus Hirschsprung; kurze Form des Morbus Hirschsprung bei ineffizienter rektaler Entleerung und Morbus Hirschsprung mit Komplikationen (Enterokolitis).

Bei der Operation ist ein Pathologe hinzuzuziehen, um sicherzustellen, dass das Stoma gut angelegt wurde. Bei der «langstreckigen Form» des Morbus Hirschsprung sollten zahlreiche Biopsien entnommen werden, u.a. auch im Appendix, und häufig ist eine Ileostomie nötig.

\section{Durchzug des Darms zum Anus}

Swenson, Duhamel und Soave haben drei gängige Operationsmethoden beschrieben. Mit diesen Methoden lassen sich, unabhängig von der Form der Erkrankung und der Länge des betroffenen Darmsegments, gleichermassen kurz- wie langfristige Ergebnisse erzielen. Zu den chirurgischen Innovationen zählen der Einsatz der Laparoskopie zur Biopsie oder intra-abdominalen Dissektion und der transanale Durchzug.

Die nachfolgend aufgeführten Punkte sind wichtig und müssen, unabhängig davon für welche Methode man sich entscheidet, besonders beachtet werden: (1) die präoperative Vorbereitung des Kolons; (2) eine perfekte histologische Analyse während des Eingriffs, um einen Durchzug in die Übergangszone zu vermeiden und (3) die antibiotische Prophylaxe.

Einige Komplikationen können schon kurz nach der Operation auftreten: (1) Die Fistelbildung an der Anastomose, die durch einen Beckenabszess manifest wird und eine Antibiose sowie häufig ein Stoma notwendig macht. (2) Die Stenose der Anastomose, die häufig auftritt, für gewöhnlich gutartig ist und gut auf eine Dilatation anspricht [45]. (3) Die Enterokolitis, bei der es sich um eine nach wie vor ernste Komplikation handelt, die bis zu zwei Jahre nach der Operation auftreten kann [46] und sich klinisch, wie bereits beschrieben, durch eine Distension des Abdomens, Diarrhoe und eine Änderung des Allgemeinbefindens manifestiert. (4) Die Hauterosion um den Anus, die in fast allen Fällen auftritt und unterschiedlich auf eine lokale Behandlung anspricht. (5) Defäkationsstörungen, die in der Langzeitnachsorge auch ohne chirurgische Komplikationen beobachtet werden [47].

\section{Operative Methoden}

Durchzug nach Duhamel. Diese Methode wurde erstmals 1956 beschrieben und beruht auf dem Erhalt des Rektums mittels retrorektalem Durchzug des normal innervierten Darms. Das Verfahren ist nach wie vor interessant, da es chirurgisch einfach zu bewerkstelligen ist und nur mit einer begrenzten Dissektion der Strukturen 


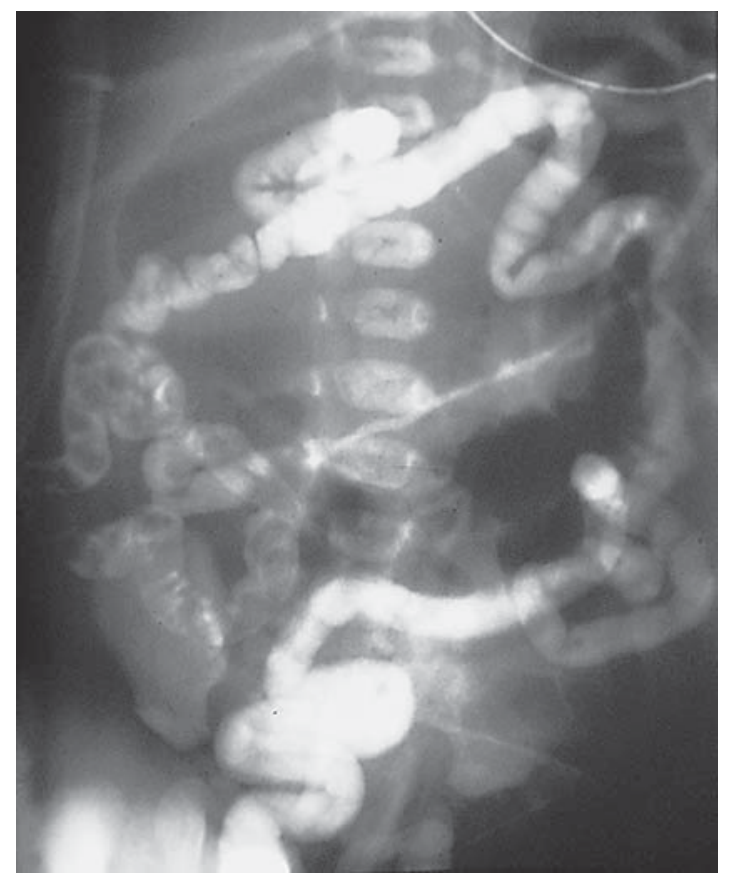

Abb. 3. Typisches Bild eines Mekonium-Ileus bei der Geburt aufgrund von Mukoviszidose.

um das Rektum (Beckennerven) einhergeht. Gleichzeitig kann mit Hilfe eines automatischen Klammernahtgeräts, z.B. einem GIA-Klammernahtgerät, die Anastomose realisiert werden. Hauptkomplikation des Duhamel-Verfahrens ist ein kolorektaler Sporn, der für ein Fäkalom verantwortlich zeichnet $[48,49]$.

Durchzug nach Swenson. Diese Methode wurde erstmals 1948 beschrieben und umfasst die vollständige Resektion des aganglionären Segments sowie eine anale Anastomose. Zu den zwei wichtigsten Komplikationen zählen die neurologische Blasenstörung (Verletzung des Beckennervs) sowie die Stenose der Anastomose.

Durchzug nach Soave. Diese Methode wurde erstmals 1963 beschrieben und besteht im Prinzip aus einem Durchzug des Kolons durch einen muskulären Rektumschlauch. Vorteil dieser Methode ist, dass keine Dissektion im Becken stattfindet. Bei einem zu langen muskulären Rektumschlauch jedoch bleibt die Obstipation als Komplikation bestehen.

Transanaler Durchzug [50]. Das Prinzip beruht auf der Realisierung der Soave-Methode, jedoch ohne Laparotomie. Diese Methode ist insbesondere bei Neugeborenen interessant, bleibt aber der kurzen Form des Morbus Hirschsprung vorbehalten [51].

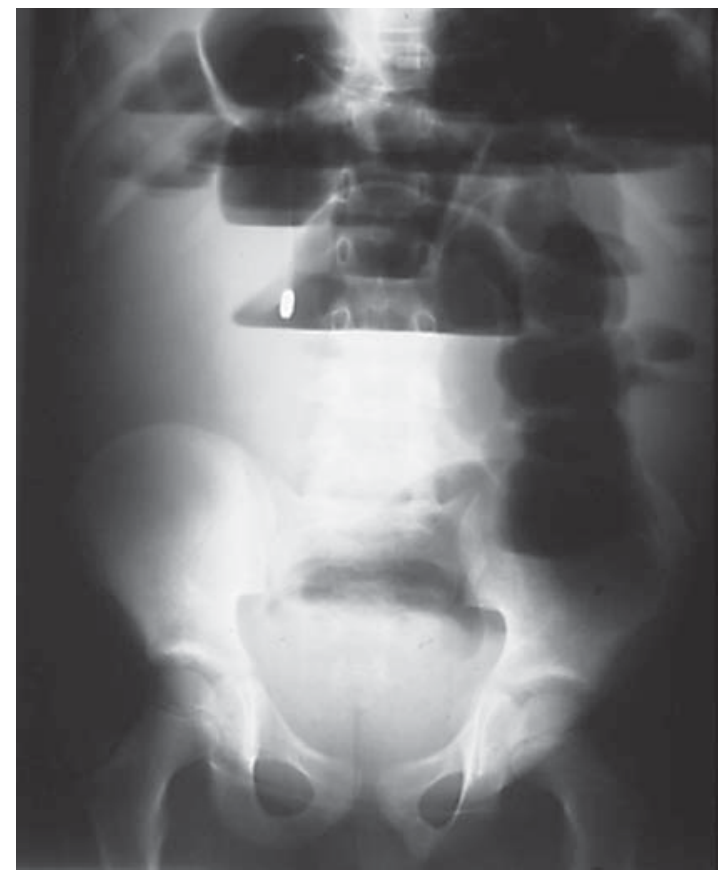

Abb. 4. Dilatation des Dünndarms bei chronischer intestinaler Pseudoobstruktion (CIPO).

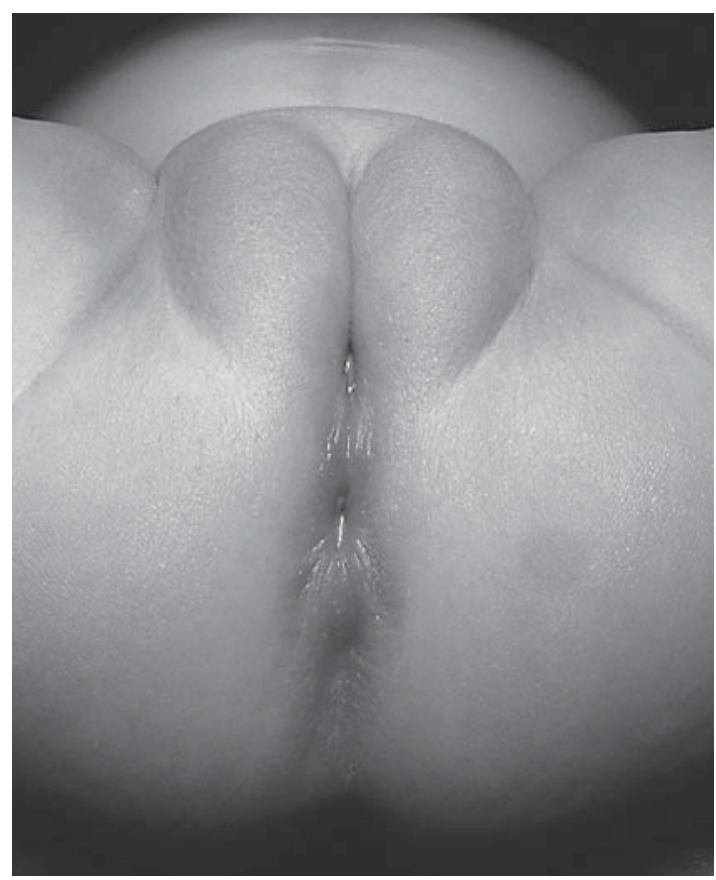

Abb. 5. Tiefe Analatresie bei einem Mädchen: Anus befindet sich an der Vulva. 
Laparoskopie. Die Laparoskopie wird zur Entnahme von Biopsien oder zur Dissektion bei den Verfahren nach Duhamel oder Swenson eingesetzt.

\section{Weitere funktionelle Obstruktionen}

Bei einem Neugeborenen mit Distension des Abdomens und verzögertem Mekoniumabgang müssen andere Ursachen der organischen oder funktionellen Obstruktion ausgeschlossen werden, z.B. die Analatresie, die Kolon- oder Ileumatresie (wird gelegentlich bei einer Morgagni-Hernie beobachtet), ein Mekonium-Ileus (bei Mukoviszidose; Abb. 3), das Small Left Colon Syndrome oder ein Mekoniumpfropf [52].

Typisch für das Small Left Colon Syndrome und den Mekoniumpfropf sind der kleine Durchmesser des Rektosigmoids und des linksseitigen Kolons bis hoch zur linken Flexur sowie des Sigmoids oder Colon descendens. Bei der Darstellung mittels Kontrastmitteleinlauf erfolgt nach rektaler Injektion der Abgang des Mekoniumpfropfs (die Spitze des Mekoniums weist häufig eine gräuliche Farbe auf, während der Rest des Mekoniums normal aussieht). Die Darmpassage ist dann völlig normal. Allerdings muss bei diesen Kindern in den ersten Lebensmonaten eine nochmalige Untersuchung und eine Rektumbiopsie durchgeführt werden, um jeglichen Verdacht auf Morbus Hirschsprung auszuräumen.

\section{Chronische intestinale Pseudoobstruktion (CIPO)}

Die chronische intestinale Pseudoobstruktion (CIPO) ist eine sehr seltene Form der Obstipation. Diese Diagnose kann bei schwerer Obstipation, die nicht auf eine medizinische Behandlung anspricht, in Betracht gezogen werden.

Typisch für die CIPO sind rezidivierende Episoden oder anhaltende Symptome und Zeichen der Darmobstruktion und die Darstellung des dilatierten Darms auf dem Röntgenbild (Abb. 4) ohne Läsion des Darmlumens, welche die Darmpassage behindert [54].

Die CIPO geht unabhängig vom histologischen Typ (Myopathie oder Neuropathie) [55] immer mit Veränderungen der kontraktilen Funktion der glatten Muskulatur einher, die zu einer Störung der Darmperistaltik und so letztendlich, aufgrund der gestörten Darmfunktion, zu Nährstoffstörungen und rezidivierender Sepsis führen. CIPO-Patienten müssen grundsätzlich auf Uropathien untersucht werden, die mit der Erkrankung einhergehen. Die klinischen Auswirkungen dieser Uropathien (Megazystis, renale Dilatation etc.) können signifikant sein und erfordern manchmal sogar eine Vesikostomie. Dieser Zusammenhang erklärt, warum eine urologische
Ultraschalluntersuchung durchgeführt werden muss, wenn die Ätiologie der Obstipation schwer zu diagnostizieren ist. Ebenso können eine Rektumbiopsie und die anorektale Manometrie notwendig sein, um das Vorhandensein von Ganglien und die Relaxation des Sphinkter internus nachzuweisen.

\section{Anorektale Malformationen}

Die anale Untersuchung bei Geburt ist bei Neugeborenen von grösster Wichtigkeit, um die rektale Permeabilität sicherzustellen. Anorektale Malformationen mit perinealer Fistel können, insbesondere bei Mädchen (Abb. 5), in den ersten Monaten oder Jahren übersehen werden, obwohl die Diagnose klinisch erfolgt. Bei diesen Fehlbildungen befindet sich der Anus im Bereich der Vulva oder des Skrotums. Der externe Sphinkter befindet sich hinter der Analfistel. Eine rektale, digitale Tastuntersuchung ist selbst mit dem kleinen Finger nicht möglich.

Wie bereits erklärt muss bei Obstipation zur Diagnosestellung stets mindestens eine fachmännische anale Untersuchung durchgeführt werden.

Die Obstipation tritt auch häufig nach Behandlung anorektaler Fehlbildungen auf [56]. Bei der hohen Analatresie, die mit Anomalien des Rückenmarks oder Sakrums einhergeht, steigt das Risiko [57]. So erreichen beispielsweise 75-85\% der Patienten mit der tiefen Form der Analatresie, aber nur 50\% der Patienten mit hoher Analatresie eine normale soziale Kontinenz. Die Behandlung der Obstipation ist bei diesen Patienten typisch und besteht aus einer medikamentösen Behandlung, Übungen zur Stärkung des Beckenbodens und in einigen Fällen chirurgischen Eingriffen (Zökostomie [58] zur anterograden Darmreinigung).

\section{Fehlbildungen des Rückenmarks oder Sakrums}

Die Obstipation kann auch neurologischen Ursprungs sein, d.h. es kann eine Verletzung des Rückenmarks (Traumata, Tumoren, Infektionen etc.) zugrunde liegen [59]. In diesen Fällen wirft die Diagnose jedoch einige Probleme auf.

Isolierte Anomalien des Sakrums oder Filum terminale manifestieren sich möglicherweise durch Obstipation. Die Untersuchung muss sich auf den Bereich des Sakrums konzentrieren (Grübchen, Lipom, Angiom, etc.). Nach Durchführung einer Röntgenuntersuchungen des Abdomens muss das Sakrum untersucht und die radiologische Untersuchung bei Neugeborenen durch Ultraschall oder anderenfalls mit Hilfe eines MRT vervollständigt werden. 
Das Currarino-Syndrom beispielsweise wird mit einem präsakralen Tumor (normalerweise einem Teratom), sakraler Dysgenesie und anorektalen Fehlbildungen (von einem fast normalen Anus bis zur hohen Analatresie) in Verbindung gebracht [60]. Erste klinische Symptome könnten Obstipation oder Harnprobleme sein.

Abschliessend lässt sich feststellen, dass die Obstipation im Kindesalter einer der häufigsten Gründe für den
Arztbesuch ist und dieser Erkrankung die verschiedensten Ätiologien zugrunde liegen können. Die Diagnose erfolgt am besten durch eine gründliche Bewertung der Anamnese und eine sorgfältige klinische Untersuchung. Weitere Untersuchungen sind zur Diagnosestellung nicht nötig und sämtliche Ermittlungen sollten nach klinischen Überlegungen durchgeführt werden.

\section{Literatur}

$\checkmark$ Constipation Guideline Committee of the $>_{14}$ Wu MJ, Chang CS, Cheng CH, et al: Colonic North American Society for Pediatric Gastroenterology, Hepatology and Nutrition: Evaluation and treatment of constipation in infants and children: recommendations of the North American Society for Pediatric Gastroenterology, Hepatology and Nutrition. J Pediatr Gastroenterol Nutr 2006;43: e1-e13.

2 van den Berg MM, Benninga MA, Di Lorenzo C: Epidemiology of childhood constipation: a systematic review. Am J Gastroenterol 2006;101:2401-2409.

-3 Khan S, Campo J, Bridge JA, et al: Long-term outcome of functional childhood constipation. Dig Dis Sci 2007;52:64-69.

-4 Singh SJ, Arbuckle S, Little D, et al: Mortality due to constipation and short-segment Hirschsprung's disease. Pediatr Surg Int 2004;20:889-891.

5 Nurko S: What's the value of diagnostic tools in defecation disorders? J Pediatr Gastroenterol Nutr 2005;41(suppl 1):S53-S55.

6 6 Drost J, Harris LA: Diagnosis and management of chronic constipation. JAAPA 2006; 19:24-29.

7 Biggs WS, Dery WH: Evaluation and treatment of constipation in infants and children. Am Fam Physician 2006;73:469-477.

8 Drossman DA, Dumitrascu DL: Rome III: New standard for functional gastrointestinal disorders. J Gastrointestin Liver Dis 2006; 15:237-241.

-9 Rubin G, Dale A: Chronic constipation in children. BMJ 2006;333:1051-1055.

10 Remes-Troche JM, Rao SS: Diagnostic testing in patients with chronic constipation. Curr Gastroenterol Rep 2006;8:416-424.

11 Safder S, Rewalt M, Elitsur Y: Digital rectal examination and the primary care physicians: a lost art? Clin Pediatr 2006;45:411414.

-12 Narbonne H, Paquis-Fluckinger V, Valero R, et al: Gastrointestinal tract symptoms in Maternally Inherited Diabetes and Deafness (MIDD). Diabetes Metab 2004;30:61-66.

13 Barrueto F Jr, Wang-Flores HH, Howland MA, et al: Acute vitamin D intoxication in a child. Pediatrics 2005;116:e453-e456. transit time in long-term dialysis patients. Am J Kidney Dis 2004;44:322-327.

15 Manz F, Wentz A: The importance of good hydration for the prevention of chronic diseases. Nutr Rev 2005;63:S2-S5.

16 Kawai M, Kubota A, Ida S, et al: Cow's milk allergy presenting Hirschsprung's diseasemimicking symptoms. Pediatr Surg Int 2005; 21:850-852.

17 Rashid M, Cranney A, Zarkadas M, et al: Celiac disease: evaluation of the diagnosis and dietary compliance in Canadian children. Pediatrics 2005;116:e754-e759.

18 Kessmann J: Hirschsprung's disease: diagnosis and management. Am Fam Physician 2006;74:1319-1322.

19 Attie T, Salomon R, Amiel J, et al: Genetics of Hirschsprung disease (in French). CR Seances Soc Biol Fil 1996;190:549-556.

20 Lyonnet S, Bolino A, Pelet A, et al: A gene for Hirschsprung disease maps to the proximal long arm of chromosome 10. Nat Genet 1993; 4:346-350.

21 Blok S, Salomon R, Pelet A, et al: Segregation of three loci explains familial and population risks in Hirschsprung disease. Nat Genet 2002;31:89-93.

22 Amiel J, Lyonnet S: Hirschsprung disease, associated syndromes, and genetics: a review. J Med Genet 2001;38:729-739.

23 Swenson O: Hirschsprung's disease: a review. Pediatrics 2002;109:914-918.

24 Reding R, de Ville de Goyet J, Gosseye S, et al: Hirschsprung's disease: a 20-year experience. J Pediatr Surg 1997;32:1221-1225.

25 Polley TZ Jr, Coran AG, Wesley JR: A tenyear experience with ninety-two cases of Hirschsprung's disease. Including sixty-seven consecutive endorectal pull-through procedures. Ann Surg 1985;202:349-355.

26 Coran AG, Teitelbaum DH: Recent advances in the management of Hirschsprung's disease. Am J Surg 2000;180:382-387.

27 Stam H, Hartman EE, Deurloo JA, et al: Young adult patients with a history of pediatric disease: impact on course of life and transition into adulthood. J Adolesc Health 2006;39:4-13.
28 Tsuji H, Spitz L, Kiely EM, et al: Management and long-term follow-up of infants with total colonic aganglionosis. J Pediatr Surg 1999; 34:158-162.

29 Jasonni V, Martucciello G: Total colonic aganglionosis. Semin Pediatr Surg 1998;7: 174-180.

30 Escobar MA, Grosfeld JL, West KW, et al: Long-term outcomes in total colonic aganglionosis: a 32-year experience. J Pediatr Surg 2005;40:955-961.

31 Elhalaby EA, Teitelbaum DH, Coran AG, Heidelberger KP: Enterocolitis associated with Hirschsprung's disease: a clinical histopathological correlative study. J Pediatr Surg 1995;30:1023-1027.

32 Elhalaby EA, Coran AG, Blane CE, et al: Enterocolitis associated with Hirschsprung's disease: a clinical-radiological characterization based on 168 patients. J Pediatr Surg 1995;30:76-83.

33 Sarioglu A, Tanyel FC, Buyukpamukcu N, Hicsonmez A: Appendiceal perforation: a potentially lethal initial mode of presentation of Hirschsprung's disease. J Pediatr Surg 1997;32:123-124.

-34 Teitelbaum DH, Coran AG: Enterocolitis. Semin Pediatr Surg 1998;7:162-169.

35 Courvoisier S, Piolat C, Durand C, et al: Pseudo-'isolated' intestinal perforation in a very low birth weight infant: exceptional presentation of Hirschsprung's disease (in French). Arch Pediatr 2006;13:1320-1322.

36 Arliss J, Holgersen LO: Neonatal appendiceal perforation and Hirschsprung's disease. J Pediatr Surg 1990;25:694-695.

37 Faure F, Panuel M, Petit PH, et al: Methodes d'explorations radiologiques de l'ano-rectum; in Le rectum pédiatrique. Monographie du college national de chirurgie pédiatrique. Montpellier, Sauramps Médical, 1996, pp 43-49.

38 Fotter R: Imaging of constipation in infants and children. Eur Radiol 1998;8:248-258.

- 39 Fondelli P, Martucciello G, Taccone A, Dodero P: The radiologic picture of total colonic aganglionosis (in Italian). Radiol Med (Torino) 1989;77:207-210. 
40 Fink RL, Roberts LJ, Scott M: The role of manometry, electromyography and radiology in the assessment of intractable constipation. Aust NZ J Surg 1992;62:959-964.

-41 Meunier P, Marechal JM, Mollard P: Accuracy of the manometric diagnosis of Hirschsprung's disease. J Pediatr Surg 1978; 13:411-415.

42 Kapur RP: Can we stop looking? Immunohistochemistry and the diagnosis of Hirschsprung disease. Am J Clin Pathol 2006;126:9-12.

-43 Matsuda H, Hirato J, Kuroiwa M, Nakazato Y: Histopathological and immunohistochemical study of the enteric innervations among various types of aganglionoses including isolated and syndromic Hirschsprung disease. Neuropathology 2006;26: $8-23$.

-44 Hartman EE, Sprangers MA, Visser MR, et al: Hirschsprung's disease: healthcare meets the needs. J Pediatr Surg 2006;41:1420 1424.

-45 Catto-Smith AG, Trajanovska M, Taylor RG: Long-term continence in patients with Hirschsprung's disease and Down syndrome. J Gastroenterol Hepatol 2006;21: $748-753$.

46 Menezes M, Puri P: Long-term outcome of patients with enterocolitis complicating Hirschsprung's disease. Pediatr Surg Int 2006;22:316-318.
47 Hyman PE: Defecation disorders after surgery for Hirschsprung's disease. J Pediatr Gastroenterol Nutr 2005;41(suppl 1):S62S63.

48 Saleh W, Rasheed K, Mohaidly MA, et al: Management of Hirschsprung's disease: a comparison of Soave's and Duhamel's pull through methods. Pediatr Surg Int 2004;20 590-593.

49 Minford JL, Ram A, Turnock RR, et al: Comparison of functional outcomes of Duhamel and transanal endorectal coloanal anastomosis for Hirschsprung's disease. J Pediatr Surg 2004;39:161-165.

50 Dasgupta R, Langer JC: Transanal pullthrough for Hirschsprung disease. Semin Pediatr Surg 2005;14:64-71.

51 Podevin G, Lardy H, Azzis O, et al: Technical problems and complications of a transanal pull-through for Hirschsprung's disease. Eur J Pediatr Surg 2006;16:104-108.

52 Yamauchi K, Kubota A, Usui N, et al: Benign transient non-organic ileus of neonates. Eur J Pediatr Surg 2002;12:168-174.

53 Cowles RA, Berdon WE, Holt PD, et al: Neonatal intestinal obstruction simulating meconium ileus in infants with long-segment intestinal aganglionosis: radiographic findings that prompt the need for rectal biopsy. Pediatr Radiol 2006;36:133-137.
54 Joly F, Amiot A, Coffin B, et al: Chronic intestinal pseudo-obstruction (in French). Gastroenterol Clin Biol 2006;30:975-985.

55 Meier-Ruge WA, Bruder E: Pathology of chronic constipation in pediatric and adult coloproctology. Pathobiology 2005;72:1-102.

56 Hartman EE, Oort FJ, Aronson DC, et al: Critical factors affecting quality of life of adult patients with anorectal malformations or Hirschsprung's disease. Am J Gastroenterol 2004;99:907-913

57 Hamid CH, Holland AJ, Martin HC: Longterm outcome of anorectal malformations: the patient perspective. Pediatr Surg Int 2006; Epub ahead of print.

-58 Yagmurlu A, Harmon CM, Georgeson KE: Laparoscopic cecostomy button placement for the management of fecal incontinence in children with Hirschsprung's disease and anorectal anomalies. Surg Endosc 2006;20: 624-627.

59 Valles M, Vidal J, Clave P, Mearin F: Bowel dysfunction in patients with motor complete spinal cord injury: clinical, neurological, and pathophysiological associations. Am J Gastroenterol 2006;101:2290-2299.

60 Kilickesmez O, Gol IH, Uzun M, Oruk C: Complete familial Currarino triad in association with Hirschsprung's disease: magnetic resonance imaging features and the spectrum of anorectal malformations. Acta Radiol 2006;47:422-426. 